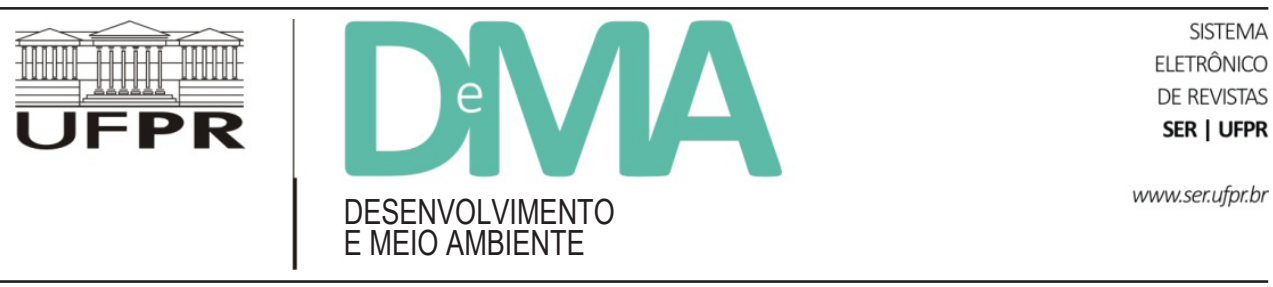

\title{
Le développement territorial durable: une nouvelle approche de développement rural ${ }^{1}$
}

\author{
O desenvolvimento territorial durável: uma nova abordagem sobre o \\ desenvolvimento rural
}

\section{Sustainable Territorial Development: a new Approach of Rural Development}

\author{
Bruno JEAN ${ }^{*}$ \\ ${ }^{1}$ Université du Québec à Rimouski (UQAR), Rimouski, Québec, Canada. \\ ${ }^{*}$ E-mail de contato/ contact: bruno_jean@uqar.ca \\ Article reçu le 20 février 2015, version finale acceptée le 15 avril 2015.
}

RÉSUMÉ: L'établissement des bases théoriques du concept de développement territorial durable sera au cœur du présent article. Il faudra d'abord rappeler l'évolution et l'actualité des hypothèses de base sur le "développement territorial" énoncées par Philippe Aydalot il y a maintenant plus d'un quart de siècle. Nous serons alors amenés à proposer une reconfiguration de ce modèle de développement, avec la notion de "développement territorial solidaire", incluant implicitement la notion du soutenabilité. Nous verrons alors comment ce modèle, misant sur le développement solidaire, peut soutenir une nouvelle perspective de développement rural, celle du développement rural durable, une perspective nécessaire pour réussir le développement des territoires ruraux. La notion de développement territorial durable peut s'enrichir de l'expérience historique comparée de développement des territoires ruraux au Nord et au Sud. Car le mal-développement régional affecte ces territoires comme les autres. Il en résulte une prise de conscience de la nécessité de penser à des systèmes ruraux durables et plus soutenables que ceux se sont mis en place avec la modernisation de ces économies rurales.

Mots clés: développement rural; territoire; ruralité; régions; solidarité; durabilité.

\footnotetext{
1 Cet article constitue une version mise à jour d'un article initial publié dans un ouvrage collectif au Québec, Voir Bruno Jean (2008), «Le développement territorial : une discipline scientifique émergente» dans Guy Massicote (sous la direction de), Sciences des territoires, Perspectives québécoises, Québec, Presses de 1'Université du Québec, pp. 283-313. Une autre version de cette article a été publié au Brésil en portugais, Voir Bruno Jean, «Do desenvolvimento regional ao desenvolvilemnto terriorial sustentavel: rumo a um desenvolvimento terrtorial solidario para um bom desenvolvimento dos territorios rurais» dans Paulo Freire Vieira, Ademir Cazella, Claire Cerdan, Jean-Paul Carriére (Orgs.). Desenvolvimento Territorial Sustentavel no Brasil, Florianopolis, APED, 2010, pp. 49-76.
} 
RESUMO: A discussão sobre as bases teóricas do conceito de desenvolvimento territorial sustentável será o objetivo principal do presente artigo. Será necessário, primeiramente, recuperar a evolução e a atualidade das hipóteses de base sobre o desenvolvimento territorial enunciadas por Philipe Aydalot há mais de um quarto de século. A partir desta revisão, proporemos uma reconfiguração deste modelo de desenvolvimento, com a noção de desenvolvimento territorial solidário, incluindo implicitamente a noção de sustentabilidade. Veremos, então, como este modelo, centrado sobre o desenvolvimento solidário, pode embasar uma nova perspectiva de desenvolvimento rural, qual seja, a de desenvolvimento rural sustentável, uma perspectiva necessária para viabilizar o desenvolvimento dos territórios rurais. A noção de desenvolvimento territorial sustentável pode ser enriquecida pela experiência histórica comparada do desenvolvimento dos territórios rurais do Norte e do Sul, já que o precário desenvolvimento regional afeta tanto uns como outros. Não seria demais afirmar que esta perspectiva resulta de uma tomada de consciência da necessidade de pensar sistemas rurais mais sustentáveis do que aqueles formados a partir da modernização dessas economias rurais.

Palavras-chave: desenvolvimento rural; território; ruralidade; regiões; solidariedade; durabilidade.

ABSTRACT: The main purpose of this paper is to fix the theoretical foundations of territorial development as an approach to understand the rural development. It was Phhilippe Aydalot, a quarter of century ago, who started to build that theory. We will then propose a reconfiguration of this development model with the concept of "inclusive territorial development", implicitly including the concept of sustainability. We will then see how this model, focusing on inclusive development, can support a new rural development perspective, the sustainable rural development, a perspective necessary for successful development of rural areas. The concept of sustainable territorial development can be enriched by the historical experience of both rural areas from the North and the South. The regional underdevelopment affects these territories like the others. We conclude on the need to think about sustainable rural systems, more sustainable than those that resulted of the modernization of the rural economies.

Keywords: rural development; territory; rurality; regions; solidarity; durability.

\section{Du développement régional au développement territorial : une nouvelle perspective}

Par un curieux glissement de perspective, la croissance économique est vite devenue synonyme du développement alors que le développement renvoie à bien plus que la croissance économique. Un économiste néoclassique au-dessus de tout soupçon, François Perroux, l'avait déjà clairement indiqué dans sa définition du développement qui reste d'ailleurs indépassée encore aujourd'hui: "Le développement est la combinaison des changements mentaux et sociaux d'une population qui la rendent apte à faire croître, cumulativement et durablement, son produit réel global" (Perroux, 1969, p. 190); et il ajoutait “Ce sont des organes d'intérêt général qui transforment la croissance d'une industrie ou d'une activité en croissance d'une nation en voie de se faire et les développements anarchiques en un développement ordonné” (Perroux, 1969, p. 205). Après des siècles de développement, et après des décennies de travail des diverses sciences du développement, il s'agit toujours d'un phénomène mystérieux et qui a donné lieu à plusieurs tentatives d'intelligibilité qui n'ont guère survécu à l'épreuve du temps.

Si les notions de territoire et de développement territorial se répandent actuellement dans le discours social comme dans le discours scientifique, c'est peut-être parce que ces notions évoquent mieux que celle de région le fait que celle-ci soit une construction sociale. Un des premiers signes de cette construction qui met en action une dynamique des acteurs sociaux sur un espace, c'est le fait qu'un territoire pour exister, doit être nommé. Une fois identifié, un territoire sert de puissant référent dans la formation de l'identité individuelle et sociale en se posant comme l'un des piliers structurants de ces identités, les autres étant sans doute les référents familiaux et professionnels. L'histoire nous montre que ce marquage territorial, s'il est influencé par une certaine lecture des réalités biophysiques, résulte aussi du 
volontarisme des collectivités humaines et de ce qu'on appelle aujourd'hui leur "projet de territoire". Les territoires sont ainsi des entités sociospatiales qui sont en lien avec les activités humaines sur un espace et ils reflètent donc une démarche de construction politique. Alain Rallet (2007, p. 80) nous le rappelait récemment en ces termes.

Développement régional, développement territorial sont deux notions distinctes. Elles renvoient à deux manières différentes d'appréhender l'espace géographique dans son rapport au développement économique (...) Le développement territorial fait référence à un espace géographique qui n'est pas donné mais construit. Construit par l'histoire, une culture et des réseaux sociaux qui en dessinent les frontières. Le contenu définit le contenant : les frontières du territoire sont les bornes (mouvantes) de réseaux socio-économiques. Là où s'éteint le réseau se termine le territoire. L'initiative ne vient pas tant d'une instance planificatrice que d'une mobilisation des forces internes.

Mais il ne s'agit pas seulement de marquer, de construire des territoires, mais de les développer. Le développement qui a été longtemps une valeur universelle, communément admise, une finalité même de toute société moderne, est aujourd'hui questionné. Il reste largement une sorte de boîte noire. On ne comprend pas trop comment il se produit car il semble être déterminé par des facteurs tant matériels qu'intangibles; on ne sait pas trop si les conséquences du développement ne sont pas plus négatives que positives. Par ailleurs, la montée en puissance de la sensibilité environnementale, avec l'émergence d'une autre notion communément admise, le développement durable, fait en sorte que le développement n'apparaît plus comme le progrès, comme la progression de l'humanité vers de meilleures conditions de vie, l'élargissement de l'expérience démocratique et l'épanouissement des cultures. Il est maintenant décrié comme une course sans fin à la croissance économique qui menacerait sérieusement la base même des sociétés humaines sur notre planète "Terre" mieux perçue dans toute sa finitude.

Le territoire est un enjeu au sens où la question régionale manifeste, au-delà de la poursuite des intérêts personnels, une quête collective de développement visant toujours un territoire donné dont l'identité et les frontières sont plus ou moins fortement établies; cet enjeu devient aussi un enjeu politique au moment où les différents territoires d'une même entité sociopolitique sont en quête d'un développement en termes de réalisation de leurs potentialités et d'un développement qui ne va pas accentuer les disparités régionales. Et le territoire est le produit du développement car ce dernier n'a de sens que s'il fournit, au-delà de la satisfaction des besoins personnels, un cadre de vie valorisant et soutenable, ce qui implique un développement territorial réussi qui correspond à la demande sociale du développement elle-même.

\section{Le développement territorial: un nouveau paradigme scientifique}

Le concept de développement territorial s'inscrit en rupture avec une tradition plus longue d'études en développement régional et il ne repose pas encore sur un corps de doctrines ou de théories fortement stabilisées. Il unit d'ailleurs deux notions - développement et territoire - qui sont des réalités que nous avons grand peine à rendre intelligibles. Mais on peut penser que le territoire ne se définit pas par son échelle mais par son mode d'organisation et par la manière selon laquelle les acteurs constitutifs des territoires s'y coordonnent (Pecqueur, 2000).

Ce processus de fabrication des territoires par des acteurs qui s'y identifient, à une échelle définie non par une instance administrative mais par un projet porté par des acteurs, s'impose comme réalité solide qui remplace de plus en plus les régulations étatiques pour "gouverner" la mutation des espaces, et notamment des espaces ruraux (Mollard \& Pecqueur, 2007).

La forme "territoire" est bien une modalité émergeante d'organisation des acteurs qui interfère puissamment dans les évolutions de la globalisation planétaire. L'orientation de PSDR n'a pas été de théoriser directement sur ce développement régional comme discipline émergeante mais (...) de faire apparaître les conditions de la constitution d'une discipline nouvelle.

En effet, tout concourre à une originalité de la démarche et de l'objet. Les méthodes sont assez variées et liées 
aux disciplines dominantes dans les recherches, mais elles convergent vers l'analyse en dynamique des comportements d'acteurs. L'objet en est le développement, c'est-à-dire une forme de dynamique non exclusivement économique dans laquelle s'intègrent des dimensions de vivre ensemble qu'attestent la présence des problématiques d'environnement, celles (trop rares) de la conquête périurbaine, des mobilités d'acteurs, des dynamiques de filières, etc. La pertinence s'appuie non seulement sur l'observation de l'homme dans son milieu, mais dans le dialogue avec les hommes définissant leur milieu de vie (Mollard \& Pecqueur, 2007, p. 17)

Cependant, on peut distinguer un certain nombre de grandes approches et de modèles d'intervention entre les deux pôles de la spécialisation et de la diversification des économies régionales, soit la théorie de la base qui mise sur le rôle structurant des débouchés extérieurs, la théorie des pôles de croissance (et maintenant des pôles de compétitivité) qui mise sur l'effet d'entraînement de villes, la théorie du commerce international qui postule la spécialisation distinctive régionale, la théorie de l'échange inégal qui tente d'expliquer les disparités de développement par le mode d'insertion dans l'économie mondiale, les théories du développement endogène (local) qui postulent la capacité des acteurs locaux à induire des dynamiques de développement plus autocentré, la théorie des milieux innovateurs, des SPL (Systèmes productifs locaux) qui met en valeur les capacités innovatrices des milieux et leur rôle dans la structuration des économies régionales. Enfin, les nouvelles théories du développement territorial mettent en valeur le rôle actif des territoires, eux-mêmes représentés non comme des supports au développement mais comme des productions sociales soutenant le développement des régions. Rappelons ici que la seule véritable théorie en matière de développement régional est celle des "pôles de croissance" énoncée par l'économiste français François Perroux dans les années cinquante et qui semble connaître un regain d'intérêt actuellement avec la notion de "pôle de compétitivité".

Le concept de développement territorial propose bien plus qu'une autre manière de rendre intelligible les réalités du développement régional et local à différentes échelles. Il s'agit d'un changement paradigmatique dans les études en développement régional ${ }^{2}$. Avec la notion de développement territorial, les sciences sociales, dans une perspective multidisciplinaire et interdisciplinaire, se donnent les moyens de reconnaître l'importance du territoire, non seulement comme une réalité biophysique bien tangible, mais aussi comme une construction sociale. Et il s'agit là, pourrions-nous dire, de l'hypothèse centrale qui fonde ce nouveau paradigme scientifique et qui nous engage à développer un nouveau savoir sur la société québécoise. Le processus de construction sociale des territoires intéresse plus particulièrement la sociologie et l'histoire alors que les dynamiques économiques spatialisées intéressent les sciences économiques au même moment où les réalités environnementales de ces territoires mobilisent la géographie et les sciences naturelles. Pour paraphraser les propos de Claude Lacour, un des chercheurs associés à l'émergence du nouveau paradigme, on peut alors dire que si "le territoire est une ressource pour le développement" (Lacour, 2000), il est aussi l'enjeu et le produit du développement.

Selon une autre figure emblématique des pionniers de la construction du champ scientifique du développement territorial, l'économiste français Philippe Aydalot (1985, p. 108), les "insuffisances de ce modèle fonctionnel classique ont amené depuis le début des années 70 à proposer un modèle de développement "territorial". Il attribue la genèse de ce nouveau modèle de développement aux travaux de John Friedmann et de Clyde Weaver et à l'impact des rapports du Club de Rome, de la Fondation suédoise Dag Hammarskjöld (la notion d'un autre développement), du concept d'éco-développement (Ignacy Sachs) et à la montée de la pensée environnementaliste. Le développement territorial implique selon Aydalot une révolution intellectuelle qui, comme Friedmann et Störh

\footnotetext{
2 Un numéro thématique intitulé "Dynamiques territoriales" (numéro 3 de 1996) de la revue Recherches sociographiques avait pris acte, il y a une dizaine d'années, de la lente maturation de ce nouveau domaine de connaissances des réalités sociales territorialisées. La longue introduction de ce numéro rappelait l'institutionnalisation de ce savoir qui va naître ici avec les travaux de Fernand Dumont et Yves Martin, fondateurs de cette revue qui a publié régulièrement des travaux sur les différentes régions du Québec. On peut décharger gratuitement tous les articles de cette revue sur le portail au www.erudit.com
} 
en montrent la voie, "associent des éléments sociaux et politiques à l'analyse économique" Il ajoute; "Un tel développement impose la rupture avec la logique fonctionnelle de l'organisation de la vie économique et propose de revenir à une vision "territoriale": c'est dans le cadre local, par la mise en valeur des ressources locales et avec la participation de la population que le développement pourra réellement répondre aux besoins de la population” Aydalot (1985, p. 109).

Au Québec, comme ailleurs dans le monde, des voies de développement et d'aménagement durables du territoire sont recherchées afin d'assurer la satisfaction des besoins et l'épanouissement des populations, tout en poursuivant des objectifs d'équité sociale, de ménagement de la nature, d'innovation économique et sociale et de participation démocratique. Dans cette perspective, la prise en compte de tous les territoires, de leur diversité et de leurs traits distinctifs, de leurs atouts et de leur complémentarité devient une impérieuse nécessité.

\section{Le développement territorial au CRDT: une perspective scientifique commune}

Pour ces chercheurs associés au Centre de recherche sur le développement territorial (CRDT), le développement territorial, considéré par plusieurs comme un paradigme en émergence, désigne à la fois un courant de recherche composite et un foisonnement d'initiatives, surtout publiques, partageant des objectifs de maîtrise des facteurs qui déterminent les performances économiques d'ensembles territoriaux plus ou moins vastes. Ces facteurs sont d'ordre économique, culturel, politique, et en même temps intrinsèquement liés aux caractéristiques (biophysiques et sociales) des territoires où interviennent des acteurs sociaux. La recherche sur le développement territorial porte sur les processus et les dynamiques territorialement ancrées de développement; elle identifie, décrit et rend intelligibles ces processus et ces dynamiques, leurs impacts pour les collectivités et les sociétés ainsi que pour les acteurs qui les habitent. Ce faisant, elle vise à comprendre comment ces acteurs interagissent avec des territoires qu'ils construisent et structurent, tout en étant structurés par eux.
Le courant du développement territorial vise à renouveler en profondeur la compréhension du rôle et de l'influence réciproque tant des structures que des acteurs sur la formation et la recomposition des espaces socio-économiques et politiques. Ce faisant, les territoires et les régions, "ces deux grands oubliés du développement" selon les mots mêmes de la Banque Mondiale, gagnent en importance, le territoire étant considéré comme "ressource active fondamentale" tout en étant aussi réhabilité par la réflexion sur le développement durable. Considéré depuis peu comme "nouvelle frontière du développement économique" par la Banque Mondiale, le développement territorial s'impose non comme un dogme ou un ensemble de certitudes, mais comme une perspective de réarticulation tant des savoirs (issus de plusieurs disciplines) que des territoires, ceci dans une direction compatible avec le progrès réel de l'humanité, supposant la répartition socialement et territorialement équitable de la richesse, la poursuite de l'expérience démocratique, la préservation et l'épanouissement des cultures.

Au sein même des pays les plus avancés, des régions entières demeurent aux prises avec des problèmes de sous-emploi, d'exode rural et de faiblesse du tissu socio-industriel. Au Québec, sept régions dites "régions-ressources" sont spécialement concernées. Ailleurs, ce sont des continents entiers ou de vastes zones surpeuplées qui s'enfoncent dans la pauvreté, en marge des circuits de la prospérité. Aussi, le CRDT entend-il porter son attention à la fois sur les régions réputées gagnantes et celles considérées perdantes ou marginales, en difficulté ou fragiles. Les traits distinctifs (notamment métropolitains, urbains, ruraux, industriels, agricoles, forestiers, miniers, maritimes, côtiers, insulaires, frontaliers, nordiques) et les dynamiques territoriales spécifiques qui caractérisent les unes et les autres retiendront aussi fortement notre attention.

\section{Le développement territorial solidaire}

Le concept de développement territorial comprend implicitement la durabilité et on peut alors soutenir que la notion de développement territorial durable est une sorte de pléonasme. Par contre, parler de développement territorial solidaire m'apparaît porteur d'une signification 
ou d'une qualification particulière du développement territorial que je propose d'utiliser pour signifier une finalité spécifique, celle de la nécessaire solidarité entre les divers groupements humains. Les communautés locales et régionales sont un type de regroupement qui peut s'organiser selon une logique individualiste, voire même égoïste ou selon une logique solidaire qui implique le partage des ressources en fonction des besoins des différentes localités et régions pour le mieux-être collectif du plus grand nombre de citoyens. Aussi, l'approche du développement territorial solidaire me semble particulièrement bien convenir pour comprendre comment devraient se construire actuellement les rapports urbains-ruraux.

Un ensemble de tendances sociales récentes, et plus particulièrement la généralisation de la sensibilité environnementale, a fait en sorte que ce qui se passe dans les campagnes est redevenu d'un grand intérêt pour les populations urbaines et pour toute la société. Les rapports urbains-ruraux traditionnels sont en train de se recomposer, certains observateurs allant jusqu'à parler d'un nouveau contrat social entre les producteurs ruraux et les consommateurs urbains. Il devient impératif d'explorer les voies par lesquelles les urbains et les ruraux, au lieu de se poser en adversaires, pourraient devenir les partenaires d'une nouvelle dynamique de développement solidaire, selon un modèle de développement territorial se construisant sur la base d'une meilleure compréhension des rapports d'interdépendances des économies rurales et urbaines, à l'heure de la nouvelle économie et du développement durable.

Le développement des vastes et nombreuses régions rurales, au Québec comme ailleurs dans le monde, ne pourra se faire qu'avec les seules ressources de ces territoires par ailleurs faiblement peuplés. Le développement de tous les territoires du Québec devient alors une question qui interpelle toute la société québécoise et non seulement les citoyens des régions en question. Il faut passer d'un certain égoïsme territorial à un développement territorial solidaire qui rend caduque la discussion sur des "redevances", soit la ré-allocation des droits perçues par l'État sur l'exploitation des ressources naturelles en fonction de leur région de provenance, une approche qui va plutôt engendrer un nouveau Québec cassé en deux: celui des villes dynamiques et celui d'une ruralité survivant chichement de redevances sur des ressources qui crée de la prospérité ailleurs. De plus, les régions rurales étant diversement dotées de ces ressources, de nouvelles disparités au sein des territoires ruraux se feraient jour.

Un large travail d'éducation socio-politique reste à faire pour montrer les avantages réciproques d'une approche partenariale en faveur de ce qui deviendrait un grand projet de société, soit le développement territorial solidaire. Cela veut dire qu'une partie des ressources fiscales des villes va servir aux gouvernements pour maintenir et développer des services dans des zones rurales à faible densité mais, en retour, ces zones pourront continuer à offrir aux populations urbaines de nombreuses aménités rurales appréciées par les citadins.

En somme, le développement territorial solidaire repose sur une meilleure compréhension des liens d'interdépendance entre les économies rurales et urbaines, sur la reconnaissance du droit des communautés rurales de se développer en mettant en valeur les ressources disponibles sur leur territoire. Il exprime notre responsabilité et notre solidarité en faveur des milieux ruraux qui rendent d'éminents services (par la production de biens publics) à toute la société. Mais dans le cas des régions rurales, l'État doit leur offrir des ressources publiques pour qu'elles continuent à réaliser une mission élargie et qui intéresse toute la société.

\section{Le développement durable}

Le développement durable fête ses 20 ans cette année et pour certains, l'heure des bilans a sonné. Si dans le grand public, développement durable rime avec protection de l'environnement, cette notion désigne, comme l'avait bien explicité Ignacy Sachs (1980), l'harmonisation des trois grandes dimensions de l'action humaine évoluant, dans nos sociétés modernes, de manière plutôt autonome et cloisonnée, soit l'environnement, l'économie et la société. Pour moi, ce qui fait la spécificité du développement durable, outre les principes d'équité sociale et inter-générationnelle, c'est cette volonté de penser un modèle de développement qui puisse rendre compatibles les opportunités économiques avec les contraintes environnementales et les attentes 
sociales, ce qu'on appelle aussi l'acceptabilité sociale qui prend une place fort importante dans la décision publique et privée ces dernières années et ce, pour une multitude de projets.

Contrairement à la vulgarisation qui en est fait, le fameux rapport sur le développement durable préparé par le premier ministre de Norvège, Mme Gro Bruntland, et publié en 1987, rappelle la nécessité d'accroître la production alimentaire pour offrir à tous une alimentation abordable. Mais il s'agit donc de civiliser l'économie en s'assurant qu'elle s'harmonise mieux avec le système des contraintes/opportunités environnementales et les attentes sociales. Nous constatons que ce qui a été le plus pris en compte dans la mise en œuvre du développement durable, c'est la dimension écologique. Alors qu'en économie, on savait mesurer la rentabilité économique, en matière d'environnement, tout était à faire car on avait très peu d'indicateurs pour qualifier l'état de l'environnement dans différents écosystèmes. Et beaucoup a été fait de sorte que, de nos jours, on peut mesurer certains développements technologiques en terme de ses effets sur l'émission des gaz à effet de serre.

Mais la mesure, avec des indicateurs sociaux validés, de l'acceptabilité sociale de telle ou telle pratique ou activité, s'est retrouvée le parent pauvre de la démarche du développement durable. La prise en compte de l'acceptabilité sociale a toutefois été mise de l'avant ces dernières années. Elle apparaît d'ailleurs comme une condition incontournable pour assurer la mise œuvre de tout projet qui a une incidence sur l'environnement, que ce soit des projets publics comme la construction d'une autoroute ou des projets privés quand un industriel veut installer une usine à quelque part. D'ailleurs, d'une certaine manière, on peut soutenir que la montée en puissance de la société civile, exigeant de participer à la prise de décision, autrement que par une démocratie représentative qui élit des décideurs pour une assez longue période, sur les interventions qui affectent son cadre de vie est une donnée toute récente et qui est directement associé avec un autre concept émergeant, celui de gouvernance.

Selon la fameuse définition de Bruntland, le développement durable consisterait en un "développement qui satisfait les besoins du présent sans compromettre la capacité des générations futures de satisfaire les leurs".
Par un glissement progressif du sens, on en est venu à penser que la responsabilité des générations présentes était de s'assurer que les générations futures puissent satisfaire leurs besoins (le plus souvent pensés comme ceux qui nous avons actuellement) alors que notre responsabilité est plutôt de s'assurer que les générations futures aient la capacité de satisfaire leurs propres besoins forcément définis par eux-mêmes. Besoin et capacité ne sont pas du même ordre, et ce sont des réalités foncièrement différentes; permettre aux générations futures de satisfaire leurs besoins, ce n'est pas du tout la même chose que leur permettre d'avoir les capacités de satisfaire les besoins qui seront les leurs. Or, cette notion de "capacité" se retrouve au centre d'une nouvelle approche appartenant au champ théorique du développement territorial (en construction), soit la théorie du développement communautaire ou du développement local qui postule que le développement repose principalement sur le "renforcement des capacités" des acteurs socio-territoriaux. Nous y reviendrons plus loin.

Le développement régional, et maintenant le développement territorial, ne sont pas d'emblée porteur de développement durable. Les régions mono-industrielles en sont un bon exemple; d'autres se questionnent sur la durabilité de certaines régions agricoles hyper-spécialisées dans l'élevage porcin intensif. On peut même observer une sorte d'asymétrie entre les démarches locales, de type Agenda 21, et les grandes conférences (Rio, Johannesburg) et les démarches des États centraux qui signent l'Accord de Kyoto sans se demander comment leurs régions participeront à cet effort collectif. Pour que les engagements pris par les États de réduire les GES (gaz à effet de serre) soient suivis d'effets réels, il faudra prendre en compte les dispositifs locaux et régionaux de gestion des ressources qui sont par ailleurs localisées. Les modes de régulation globaux doivent donc se décentraliser; et on retrouve ici un autre argument pour les régionalistes en quête de légitimité.

D'autres encore comme Olivier Godard (2007) pensent que le développement durable implique que les acteurs de développement "doivent apprendre à jouer de manière subtile de cette pluralité d'espaces de développement". Le lien entre le développement territorial et le développement durable passerait donc par cette idée de l'articulation de la pluralité d'espaces de développement 
qui permet à un territoire donné d'atteindre un état de résilience. Cette notion désigne la capacité à retrouver un fonctionnement et un développement normal après un stress, une perturbation, un changement important. Mais la résilience est certainement en lien avec la faculté d'adaptation qui implique une capacité d'ajustement, voire de reconversion, et certainement une capacité d'anticipation proactive des évolutions. Bien qu'un tel lien ne soit pas, à notre connaissance, identifié dans la littérature, le développement durable implique une bonne capacité d'adaptation des systèmes socio-économiques aux inévitables perturbations car un système peu flexible ne serait certainement pas durable dans le temps.

Comme plusieurs le constatent, les grands principes du développement durable et ceux qui portent les nouvelles théories du développement territorial ne sont pas si éloignées sur le plan épistémologique et se rejoignent sur un point fondamental, soit la reconnaissance des capacités des acteurs sociaux qui construisent quotidiennement des territoires où s'articulent une pluralité de logiques de développement.

\section{Le développement rural durable et sa représentation dans un modèle causal}

L'application des principes du développement durable aux milieux ruraux est d'autant plus intéressante que la durabilité de plusieurs régions et communautés rurales est menacée par l'actuelle recomposition des territoires en lien avec la mondialisation. Et d'autre part, la théorie du développement territorial esquissé par Aydalot est cohérente avec la démarche du développement durable surtout avec une approche territoriale où les choix de développement sont pris localement avec les acteurs concernés. Aussi, on peut alors affirmer que le développement rural durable est une approche globale, territoriale, et intégré du développement des collectivités rurales qui repose sur une triple harmonisation entre les opportunités économiques, les contraintes environnementales et l'acceptabilité sociale. La mise en œuvre d'une démarche de développement rural durable devrait aussi assurer, pour plusieurs territoires en dévitalisation, une revitalisation rurale soutenable qui repose sur la vitalité sociale des communautés rurales, la viabilité économique des économies locales et la validité écologique des systèmes ruraux.

Des territoires ruraux inscrits dans une perspective de développement territorial durable ressembleraient à quoi? Premièrement, des territoires avec des écosystèmes en santé, soit avec l'air et de l'eau pure, un paysage agréable et un patrimoine naturel et bâti bien entretenu. Deuxièmement, un territoire manifestant une viabilité économique, c'est-à-dire des territoires où on observe une croissance des capacités d'entreprenariat, une diversification économique et une réduction de la pauvreté. Troisièmement, des territoires jouissant d'une plus grande équité sociale avec l'arrêt de la migration rurale, notamment des jeunes, avec une plus grande inclusion sociale des minorités et des femmes, avec une plus grande accessibilité aux services.

Dans le cadre de la démarche d'évaluation de la première politique rurale du gouvernement du Québec que nous avons réalisé avec plusieurs autres collègues ${ }^{3}$, nous avons été amenés à penser la dynamique d'un développement durable des communautés rurales en construisant un modèle causal qui identifie les facteurs ou les variables, et leurs interrelations, et qui agissent pour susciter ou un non un développement territorial durable dans le cas des espaces ruraux. Ce modèle nous permettait de situer le point d'entrée de cette politique publique sur la ruralité québécoise dans la dynamique de ce modèle et on voit bien qu'elle intervient au niveau de ce que nous avons appelé la mobilisation et qui au cœur de notre modèle causal qui repose sur un paradigme scientifique qui met l'accent sur l'approche souvent appelée celle du "renforcement des capacités" (capacity-building). Cette approche postule que le développement passe par un processus d'apprentissage social où on apprend à le faire en le faisant (learning by doing), de là, cette notion de "projets" aussi au cœur de la théorie (projets de territoire). Dans les lignes qui suivent, nous allons commenter brièvement ce modèle reproduit ici.

\footnotetext{
3 Voir: Bruno Jean, Patrice Leblanc, Danielle Lafontaine, Richard Marceau, et al., Le modèle et les indicateurs de suivi et d'évaluation du dispositif des Pactes ruraux, Rimouski, CRDT, Février 2007, 108 p. et La mise en application et l'évaluation du dispositif du Pacte rural, Rapport de recherche 2, CRDT, Université du Québec à Rimouski. On peut consulter le rapport complet en ligne: http://www.fqrsc.gouv.qc.ca/
} 
Le processus du développement rural peut être modélisé en identifiant les liens de causalité entre cinq grands groupes de variables, soit quatre variables intermédiaires (CAPITAUX, USAGES, MOBILISATION, DÉVELOPPEMENT) et une variable dépendante (COMMUNAUTÉS RURALES DURABLES). Dans un modèle, la variable dépendante, c'est celle qu'il faut expliquer, qui nous intéresse en ce sens que le modèle fait une hypothèse sur l'effet des facteurs sur la variance de cette variable ou sur les facteurs qui sont des variables indépendantes en amont, si on peut dire.

Dans ce cas-ci, le modèle fait aussi une hypothèse sur l'effet attendu des Pactes ruraux dans cette dynamique de développement des milieux ruraux. Tenant compte de notre compréhension des intentions énoncées dans la Politique Nationale de la ruralité (PNR), il était évident que ces intentions se situaient au niveau d'interventions susceptibles d'accroître les "capacités de développement" de ces milieux en intervenant sur les capacités de mobilisation par un plus grand engagement citoyen, une meilleure gouvernance rurale et la mise en action des acteurs locaux dans des "Actions innovantes".

\section{Le modèle causal du développement rural}

Dans le modèle théorique du développement rural proposé ici, le "développement" est une variable intermédiaire qui est déterminée principalement par la "mobilisation" et qui détermine à son tour la durabilité des communautés rurales, la variable indépendante finale du modèle. Dans le modèle, ce qui nous intéresse, ce n'est pas tant le développement en soi qui renvoie plutôt à la variable finale mais à la mise en place ou non ou à des degrés divers de processus observables de renforcement des capacités de développement dans les communautés rurales et d'une dynamique de développement se révélant par des "actions de développement". Pour moi, la notion de renforcement des capacités de développement (Jean, 2003) désigne un processus par lequel les individus, les groupes, les organisations et les sociétés renforcent leurs habilités à identifier et à relever les défis du développement sur une base durable. Pour mesurer le renforcement des capacités, quatre grands groupes d'indicateurs ont été identifiés: 1) Capacité des acteurs locaux à diagnos- tiquer l'état de situation du développement économique et social de leur communauté; 2) Capacité des acteurs locaux à mettre en place une organisation territoriale de développement; représentative et efficace; 4) Capacité des acteurs locaux à élaborer un plan stratégique de développement local; 5) Capacité des acteurs locaux à évaluer objectivement les résultats et à faire mettre en place une planification itérative.

Si le dispositif des Pactes ruraux de la PNR intervient sur ce groupe de variables indépendantes du modèle (MOBILISATION, DÉVELOPPEMENT), il arrive dans des milieux qui se différencient selon ce que nous avons appelé la variable "CAPITAUX", soit leur dotation initiale différente et divers "atouts" (leurs "assets" comme on dirait en anglais), leurs avoirs ou leurs actifs qui ne sont pas seulement financiers mais aussi et d'abord humains, mais également naturels, physiques, "usages" il en est fait. Par exemple, le modèle prédit qu'un trop fort exode des jeunes (capital humain) ne va pas contribuer positivement au développement de communautés rurales durables; de même, le modèle prédit qu'une sur-exploitation de la forêt, au-delà de sa capacité productive, aura le modèle effet sur le phénomène qui nous intéresse, la viabilité et la durabilité des communautés rurales.

Dans le modèle, la mobilisation ne produit pas directement le développement. Seule une mobilisation qui est agissante, qui initie des projets de territoires si on peut dire, qui favorise un apprentissage social est susceptible susciter du "Développement". Pour nous, il y a développement lorsqu'il y a augmentation observable des "capacités de développement" ainsi qu'une croissance des "Actions de développement" ou la mise en place d'une dynamique de développement, également mesurable par des indicateurs objectivement observables. On aura vite compris ici que si ce modèle est à la fois bien fondée théoriquement, en prenant en compte l'état des connaissances sur la ruralité et les théories du développement et en postulant l'efficience des approches ascendantes de développement. Si ce modèle est bien celui qui sous-jacent à la PNR, il reste un défi de taille, soit identifier les bons indicateurs pour la mesure de toutes ces variables.

Cela commanderait ici un programme de recherche de plusieurs années pour valider ces indicateurs. L'option 


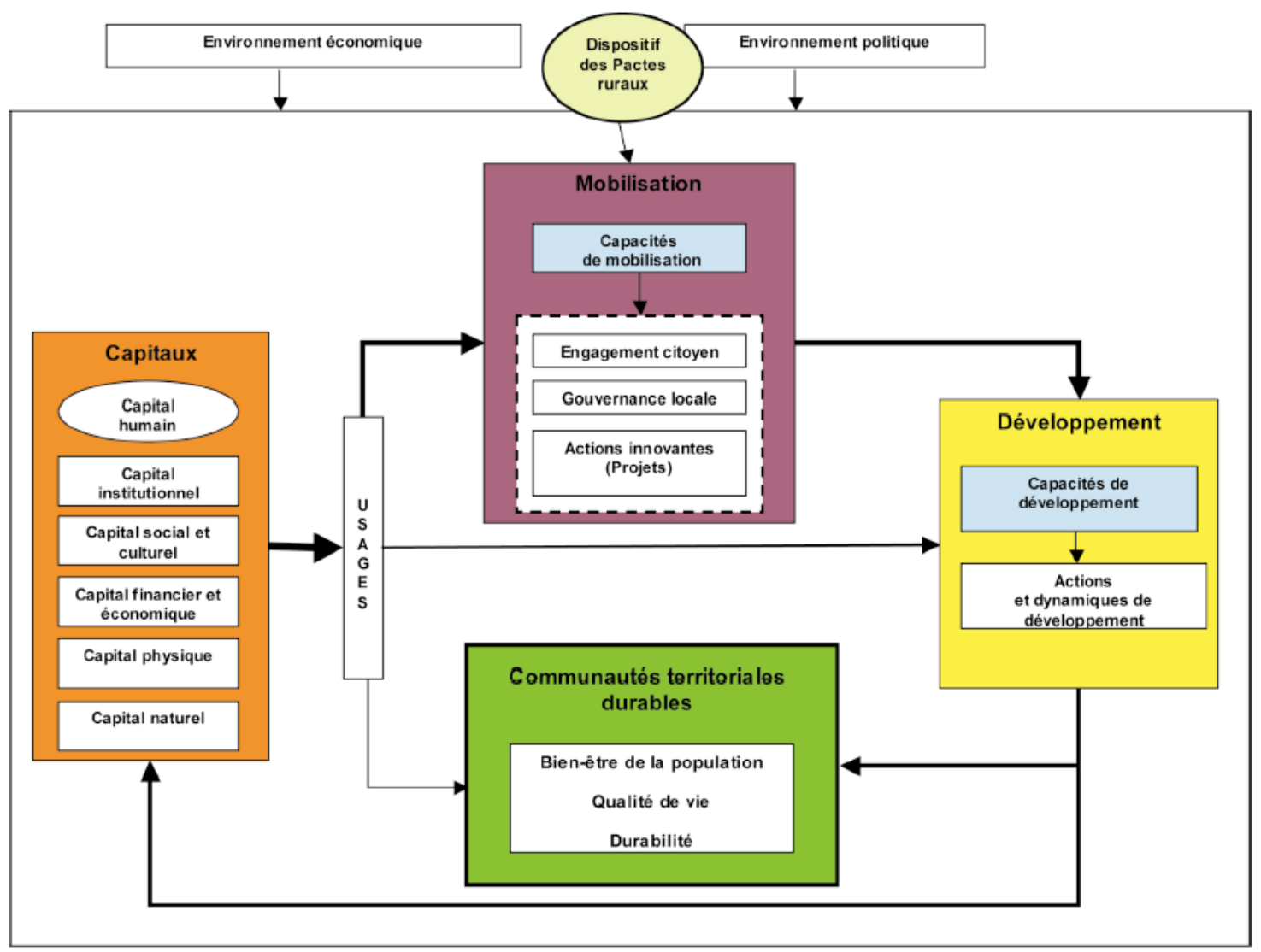

FIGURE 1 - Le modèle causal de développement rural.

retenue ici a été de nous concentrer sur les variables sur lesquelles le dispositif des Pactes ruraux était supposé avec un effet, donc celles contenues dans la grande variable "mobilisation". Par mobilisation, nous entendons ici l'aptitude d'une communauté donnée à stimuler la participation des différentes catégories de citoyens qui la composent en vue de déterminer des buts communs et des actions collectives innovantes, de renforcer les réseaux de communication interne et de mettre en place une gouvernance efficiente capable de traiter les conflits. Ainsi définie, il y a trois grandes sous-dimensions qui sont sous-jacentes à ce concept de mobilisation, soit l'engagement citoyen, la gouvernance et la mise en oeuvre d'actions innovantes. Pour opérationnaliser de telles notions, il nous est apparu qu'il était possible d'identifier des indicateurs qui concernent: 1) la capacité des acteurs locaux à stimuler la participation civique et l'engagement social; 2) la capacité des acteurs locaux à faire naître une volonté commune d'intervenir; 3) la capacité des acteurs locaux à renforcer l'esprit d'entreprise et d'entraide entre les membres de la collectivité.

Par ailleurs, au terme d'un horizon d'au moins dix $a^{4}{ }^{4}$, on pourra mesurer si les effets postulés par ce modèle causal se produisent dans la réalité. Il conviendrait

${ }_{4}$ Pour ces politiques publiques visant le renforcement des capacités, on pense maintenant qu'il faut au moins dix ans pour observer des changements durables, que ce soit avec les programmes LEADER en Europe ou les Rural Partnerships aux USA ou le programme Community Futures (SADC) au Canada. Voir: J. N. Reid and C. Flora, "Advancing Knowledge and capacity for Community-led Development” dans Participatory Governance, edited by W. Robert Lovan, M. Murray, and R. Scaffer, Ashgate, Londres, 2003. 
alors que voir si les communautés rurales, notamment celles qui ont été identifiés comme défavorisées, ont changé de trajectoire de développement en devenant des COMMUNAUTÉS TERRITORIALES DURABLES. On peut penser que le retournement des tendances démographiques régressives vers la stabilité ou même une croissance, même légère, est un signe probant des effets positifs des Pactes ruraux. Mais nous pensons également que cette grande variable dépendante de notre modèle causal se décompose en trois dimensions: 1) le bien-être de la population qui se mesure généralement par le niveau de revenu individuel et son écart à la moyenne et qui devrait se réduire; 2) la qualité de vie qui pourrait se mesurer par des indicateurs de santé publique, comme l'incidence des maladies coronariennes ou autres et; 3 ) la durabilité des communautés qui peut se mesurer par des indicateurs de participation sociale et de mesure de qualité de l'environnement.

\section{Conclusion: pour un développement territorial durable, solidaire et approprié pour réussir le développement des territoires ruraux}

Le développement territorial durable, solidaire et approprié des communautés rurales suppose de réunir plusieurs conditions gagnantes dont chacune est nécessaire mais non suffisante. On peut parler des conditions gagnantes pour contrer les processus de dévitalisation rurale ${ }^{5}$. Différents travaux de recherche m'ont amené à conclusion qu'une dizaine de conditions étaient nécessaire pour réussir le développement des territoires ruraux; ces conditions à réunir sont autant d'enjeux majeurs pour ces territoires. Ces dix conditions gagnantes peuvent être énoncées de la manière suivante : reconnaître qu'on peut apprendre de la ruralité; protéger la socio-diversité à laquelle contribue la ruralité; favoriser une approche de développement rural durable; reconnaître la multifonctionnalité de la nouvelle économie rurale; mobiliser le capital social et les infrastructures sociales entrepreneuriales; soutenir le développement des capacités des communautés rurales; soutenir l'émergence d'une nouvelle gouvernance rurale citoyenne; rendre intelligible l'interdépendance rurale-urbaine; soutenir une nouvelle solidarité rurale-urbaine par une taxe pédagogique; mettre en œuvre une politique publique appropriée. Le principal postulat derrière l'énoncé de ces conditions, c'est que la responsabilité du développement appartient aux ruraux et à leurs communautés. Un tel postulat implique un sérieux changement de mentalité dans des territoires où des décennies de l'État-Providence a généré une attitude de dépendance et attentiste et où on est déresponsabilisé face à l'aménagement de son propre avenir.

Les bases théoriques du développement territorial explicitées ici sont telles que le qualificatif de "territorial" semble superflu quand on sait que le développement, par définition si on peut dire, est territorialisé. Car le développement fonctionnel ou sectoriel est daté historiquement. Il s'est imposé avec l'État modernisateur des temps justement appelés "modernes". Cette rationalité modernisante s'opposait fortement à tous ces liens communautaires, socio-territoriaux, qui fondaient les particularismes locaux, typiques des sociétés rurales, pour faire accéder ces gens à l'universalisme culturel et aux bienfaits de la civilisation.

Comme le montre l'expérience africaine de développement rural, les projets de développement qui ont du succès sont ceux qui sont capables de ne pas détruire mais au contraire d'intégrer le passé dans le présent et le futur. Au vieux débat entre le respect de la tradition et la recherche de modernité, il ne faut pas choisir entre l'un ou l'autre, mais respecter les traditions et les perpétuer mais tout en étant capables d'innovation car ce sont les innovations techniques, sociales et institutionnelles qui assurent l'avenir. Mais l'aménagement du futur n'est possible que si la citoyenneté est assurée. Ce prérequis est difficile à comprendre quand on a grandit dans un pays qui respecte ce principe de base mais on le comprend mieux en prenant en compte une expérience comme celle des paysans sans terre du Brésil.

C'est en Afrique que j'ai pris conscience du processus d'appropriation des projets de développement. L'enjeu majeur, pour un organisme donateur, était de

\footnotetext{
5 Voir Bruno Jean, "Réussir le développement des communautés rurales: dix conditions gagnantes" dans Organisations \& Territoires, 12(2), 19-31, 2003 (Université du Québec à Chicoutimi, ISSN 1493-8871).
} 
s'assurer de la reprise des équipements mis en place (ou des institutions) par les destinataires de cette aide, les communautés locales, lorsque l'assistance technique étrangère serait partie. La notion d'appropriation du développement a alors été popularisée par les experts des institutions internationales de développement dans le cadre des nombreuses études d'évaluation des projets dits de développement dans les pays non-développés. Le concept d'appropriation s'inscrit dans ces nouvelles approches qui misent sur l'autodétermination des communautés territoriales. Il trouve sa double origine tant en Afrique, avec l'animation rurale, qu'en Amérique avec le community development. Il s'agit de construire des projets de développement qui répondent à des besoins des communautés locales, à leurs attentes et qui s'inscrivent harmonieusement dans leur réalité socio-historique profonde.

Parler de développement approprié, c'est désigner ce que plusieurs appellent le développement territorialisé et le développement durable. Mais appropriation du développement et développement approprié ne sont pas synonymes; un large fossé épistémologique existe entre les deux. Dans le premier cas, le développement repose sur un ensemble connu d'atouts ou d'actifs (ressources, savoirs-faire, techniques, institutions, etc.) qui sont transférés par l'assistance technique aux populations ainsi assistées. L'évaluation consiste alors à mesurer le degré d'appropriation des actions de développement. Dans le second cas, la formule implique une interrogation implicite : approprié oui, mais approprié à qui... ou pour qui? Le développement existe d'abord pour le

\section{Bibliographie}

Aydalot, P. Économie régionale et urbaine. Paris: Économica, 1985.

Godard, O. Le développement régional au développement durable: tensions et articulations. In: Mollard, A. et al. Territoires et enjeux du développement régional. Versailles: Éditions Quae, 2007, p. 83-96.

Jean, B. Réussir le développement des communautés rurales: dix conditions gagnantes. Organisations \& Territoires, 12(2), 19-31, 2003. vrai monde, comme on dirait dans la langue québécoise, pour des personnes, des communautés humaines. Si le développement est fait pour et par le monde, les acteurs sociaux qui sont responsables de la conception des actions de développement et ceux à qui ces actions sont destinées doivent être les même acteurs. Ainsi, pas de développement approprié sans un long travail avec les populations pour arriver à une vision commune et partagée du diagnostic de la situation et des moyens à prendre pour aménager durablement cet avenir planifié mutuellement. Ainsi définie, la notion de développement approprié m'apparaît tout aussi pertinente que les diverses notions dérivant du champ sémantique du développement local et, plus récemment, de celui du développement durable.

Le développement territorial implique aussi que chaque territoire construise, par une démarche interne, son propre modèle spécifique de développement. Car ce qui a réussi sur un territoire donné à un moment donné peut fort bien ne pas réussir dans un autre territoire. La non-reproductibilité des modèles de développement impose la reconnaissance d'une autre valeur, celle la créativité qui doit s'ajouter à celles de la responsabilité et de la solidarité que nous avons évoquées ici. Réussir le développement territorial suppose un processus d'apprentissage social du développement, c'est-à-dire le développement d'une compétence citoyenne dans le cadre d'un modèle de développement, qui est aussi un modèle de développement social visant une participation sociale partagée entre la revendication des droits et l'apprentissage des responsabilités.
Lacour, C. Les territoires, nouvelles ressources du développement. In: INRA. Recherches pour et sur le développement territorial, Symposium de Montpellier, 11-12 janvier, 2000.

Mollard, A; Pecqueur, B. Le développement régional: enjeux de recherche et d'acteurs. In: Mollard, A. et al. Territoires et enjeux du développement régional, Versailles: Éditions Quae, 2007, p. 15-35.

Pecqueur, B. Le développement local: pour une économie des territoires. Paris: Éditions Syros, 2000. 
Perroux, F. L'économie du XXe siècle. Paris: Presses universitaires de France, 1969.

Rallet, A. Commentaires du texte d'Oliver Crevoisier. In : Mollard, A. et al. Territoires etjeux du développement régional. Versailles: Éditions Quae, 2007, p. 80.
Recherches Sociographiques. Le développement territorial. Numéro thématique sous la direction de Bruno Jean, Vol. XLVII, n. 3, 2006.

Sachs, I. Stratégies de l'écodéveloppement. Paris: Éditions Économie et Humanisme, 1980. 